Rechtsmedizin $2011 \cdot 21: 177-178$

DOI 10.1007/s00194-011-0768-7

Online publiziert: 18. Mai 2011

(c) Springer-Verlag 2011

\author{
H. Bratzke ${ }^{1} \cdot$ K. Püschel ${ }^{2}$ \\ ${ }^{1}$ Institut für Rechtsmedizin, Klinikum der Johann \\ Wolfgang Goethe-Universität, Frankfurt a. M. \\ ${ }^{2}$ Institut für Rechtsmedizin, Universitätsklinikum Hamburg-Eppendorf, Hamburg
}

\section{Medikolegale Begutachtung des Schädel-Hirn-Traumas}

Im Hinblick auf traumatologische, biomechanische und rekonstruktiv gutachterliche Aspekte von Gewalteinwirkungen kommt den intrakraniellen Verletzungen „zentrale“ Bedeutung zu, da sie ca. $15 \%$ des Sektionsguts betreffen. Dieser hohe Stellenwert des Schädel-Hirn-Traumas steht in einem gewissen Gegensatz zu den wissenschaftlichen Schwerpunktsetzungen in unserem Fach; vergleichsweise wenige Fachvertreter bzw. rechtsmedizinische Institute haben hier in der Vergangenheit nachhaltig Zeichen gesetzt.

Zum Teil dürfte dies damit zusammenhängen, dass sich die Neuropathologie als eigenständiges Fachgebiet herausgebildet hat, wobei sich die Fachvertreter durchaus als Zusatzgutachter in medikolegalen Fragen zu Wort gemeldet und bewährt haben. Andererseits sind die Sektion und die mit tödlichen Gehirnverletzungen zusammenhängenden traumatomechanischen Fragestellungen eine Domäne der Rechtsmedizin und als „Alleinstellungsmerkmal“ von besonderer Bedeutung. Auch bei bester Kooperation zwischen Rechtsmedizin und Neuropathologie kommt es entscheidend darauf an, dass bei der Sektion die angemessene Technik zur Untersuchung und zur Dokumentation der entscheidenden Befunde eingesetzt wird und dass die Zusammenhangsbegutachtung beim Rechtsmediziner verbleibt.

\section{Die Zusammenhangsbe- gutachtung muss beim Rechtsmediziner verbleiben}

Wichtige Akzente hat die „Berliner Schule" gesetzt (Krauland, Bratzke, Maxeiner), nachdem wesentliche biomechanische Grundlagen von Sellier u. Unterharnscheidt [1] erarbeitet und Anfang der 9oer Jahre im vierbändigen Handbuch aus diesem Verlag zusammenfassend dargestellt wurden. Hinzu kommen die sehr umfassenden Arbeiten von Oehmichen, speziell im Hinblick auf moderne histologische und immunhistologische Techniken.

Die Autoren, die sich in neuerer Zeit der Neuropathologie/Traumatologie stärker zugewendet haben, kommen im vorliegenden Heft der Zeitschrift Rechtsmedizin zu Wort. Dabei wurden zuletzt die moderne bildgebende Technik postmortal weitergehend genutzt (Computertomographie, Magnetresonanztomographie; hierzu Büttner et al.) und besonders gefährdete Personengruppen (z. B. Kindesalter; Büttner et al.) sowie spezielle äußere Rahmenbedingungen (Sport; Matschke et al.) beleuchtet. Dass nicht selten primär auch innere Hirnstrukturen verletzt werden, haben Maxeiner et al. herausgearbeitet. Rothschild et al. analysierten die spezielle Traumatogenese beim Kopfschuss. Das Gehirn ist physikalisch bei wundballistischer Betrachtung als ausgesprochen unelastisch und kaum komprimierbar an- zusehen. Schließlich wenden sich Zindler et al. nochmals dem Phänomen der Contrecoup-Läsion zu, die am ehesten einem Kontakttrauma mit vorwiegend translatorischer Komponente zuzuordnen ist.

Subtile Kenntnisse, eine sorgfältige, insbesondere gezielte und schonende Präparationstechnik (u. U. auch mit speziellen Zugangswegen und Schnitttechniken), umfangreiche traumatomechanische Kenntnisse sowie gutachterliche Erfahrungen sind Voraussetzung für eine überzeugende Bearbeitung und Begutachtung dieser Fälle, spezielle mikroskopische (histologische, histochemische) Kenntnisse im Hinblick auf die topografischen Prädilektionsstellen unabdingbar.

Mit dem hier vorliegenden Schwerpunktheft erinnern wir an unseren engagierten Kollegen Helmuth Maxeiner, der 2009 viel zu früh von uns gegangen ist: $\mathrm{Er}$ war im positiven Sinn ein unbequemer, dabei sehr kreativer Geist, der sich stets durch eine gezielte Präparationstechnik und besonders sorgfältige sowie kritische makroskopische und mikroskopische Befunddeutung ausgezeichnet hat.

Angesichts zunehmender Verfeinerung postmortaler bildgebender Verfahren bleibt die sorgfältige Technik der Präparation des knöchernen Schädels, der Gefäßaufzweigungen und der Lokalisation von Verletzungen im Gehirn im Rahmen der Sektion nach wie vor unabdingbare Voraussetzung zur Rekonstrukti- 
on und Begutachtung des Schädel-HirnTraumas. Art, Ausmaß, Richtung, Reihenfolge, Häufigkeit, zeitliche Abfolge von Läsionen und die Abgrenzung gegenüber krankhaften Veränderungen können nur durch direkte Untersuchung geklärt werden.

\section{() Der Verzicht auf ein Alleinstel- lungsmerkmal bedeutet eine Verarmung der Rechtsmedizin}

Gerade den jüngeren Kollegen wollen wir nahebringen, dass mit dem Wissen in der Neurotraumatologie auch der Zweifel wächst. Ein vertieftes neurotraumatologisches Verständnis ist für den Rechtsmediziner unabdingbare Grundlage der fundierten Begutachtung und kann nicht durch eine Verfahrensweise ersetzt werden, bei der der Rechtsmediziner sich auf die äußerliche Untersuchung des Gehirns beschränkt und die Gutachtenerstattung anderen überträgt. Der Verzicht auf ein Alleinstellungsmerkmal bedeutet eine Verarmung der Rechtsmedizin und gefährdet das Fach in seinen Grundfesten.
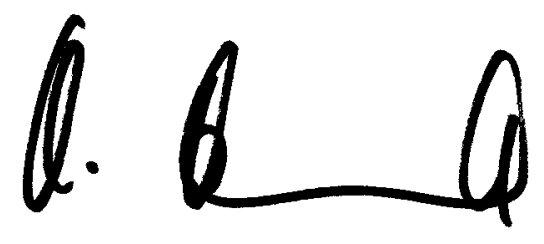

Hansjürgen Bratzke
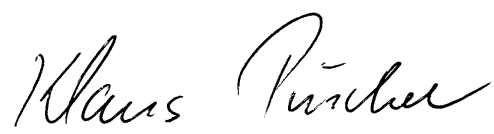

Klaus Püschel

\section{Korrespondenzadressen}

\section{Prof. Dr. H. Bratzke}

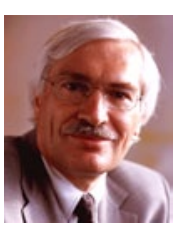

Institut für Rechtsmedizin, Klinikum der Johann Wolfgang Goethe-Universität Kennedyallee 104, 60596 Frankfurt a. M. bratzke@em.uni-frankfurt.de

\section{Prof. Dr. K. Püschel}

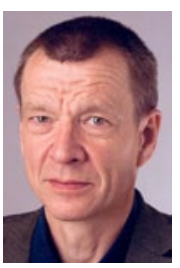

Institut für Rechtsmedizin, Universitätsklinikum Hamburg-Eppendorf Butenfeld 34, 22529 Hamburg pueschel@uke.de

Interessenkonflikt. Die Autoren geben an, dass kein Interessenkonflikt besteht.

\section{Literatur}

1. Sellier K, Unterharnscheidt F (1963) Kavitationstheorie beim Contrecoup-Phänomen. In: Sellier K Unterharnscheidt F (Hrsg) Mechanik und Pathomorphologie der Hirnschäden nach stumpfer Gewalteinwirkung auf den Schädel. Beiheft 76 zur Monatsschr Unfallheilkunde. Springer, Berlin Heidelberg New York Tokio

\section{Adrian Wallwork English for Presentations at International Conferences}

Heidelberg: Springer 2010, $196 \mathrm{~S}$., (ISBN 978-1-4419-6590-5), 26.70 EUR

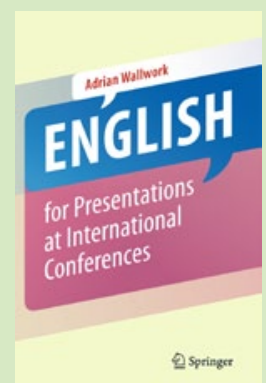

Der Autor möchte sein Werk als Leitfaden und Nachschlagewerk für die Erstellung von (englischsprachigen) Vorträgen auf internationalen Kongressen verstanden wissen. Dabei liegt der

Schwerpunkt auf der sprachlichen Seite und nicht der technischen oder grafischen Ausgestaltung der Präsentationen. Bezüglich der Zielgruppe gelingt ein Spagat von dem Anfänger in wissenschaftlichen Vorträgen für den Englisch keine Muttersprache ist bis hin zum erfahrenen Wissenschaftler, der als „,native speaker" auf die Welt gekommen ist. So gibt es banale Hinweise über Intentionen eines wissenschaftlichen Vortrags genauso wie Tipps für Fortgeschrittene. Die inhaltlichen, didaktischen und organisatorischen Informationen sind keineswegs auf den Einsatz in der englischen Sprache beschränkt und können auch für deutschsprachige Vorträge auf unseren Frühjahrs- oder Jahrestagungen verwendet werden. Beispielsweise ist das Kapitel zum Umgang mit der Nervosität („Handling your Nerves") natürlich unabhängig von der tatsächlichen Sprache. Wenngleich der Hinweis für die „Fremdsprachler" kommt, dass im Auditorium keine Englischlehrer sitzen sondern Wissenschaftler, die an den präsentierten Ergebnissen interessiert sind. Dennoch werden die Kolleginnen und Kollegen am meisten von dem Buch profitieren, die sich auf englischsprachige Vorträge vorbereiten wollen. Negativ fallen die Druckqualität und die zu kleine Schrift auf. Offenbar wurde das für DIN A4 konzipierte Manuskript auf ein Zwischenformat verkleinert. 